\title{
LA SIGNIFICACIÓN ONTOLÓGICA DE LA PRIMORDIALIDAD DE LA SUSTANCIA EN ARISTÓTELES
}

Luis Fernando Mendoza Martínez*

RESUMEN: Aristóteles, en el libro Z de la Metafísica, busca determinar el sentido en el que la sustancia se constituye como ente primordial. Los criterios de dicha primordialidad son: en cuanto al concepto o enunciado, en cuanto al conocimiento y en cuanto al tiempo. De las dos primeras formas de primordialidad de la sustancia ofrece el Estagirita algunas pautas para comprenderla, pero acerca de la primordialidad en cuanto al tiempo el asunto es más oscuro. En este trabajo se arrojan luces a la cuestión de en qué consiste la primordialidad en cuanto al tiempo.

yose

THE ONTOLOGICAL MEANING OF ARISTOTLE'S PRIMORDIALISM OF SUBSTANCES

ABSTRACT: Aristotle endeavors to learn how substances become primary entities in his Metaphysics book Zeta. He provides three criteria for such primordialism, namely that of concept or statement, that of knowledge, and time. Of the first two, the Stagiritte provides some clues to their understanding, but regarding the third the matter is less clear. In this article, we will shed some light on the primordialism with respect to time.

PALABRAS CLAVE: Sustancia, primordialidad, tiempo, movimiento.

KEY WORDS: Substance, primordialism, time, movement.

RECEPCIÓN: 27 de noviembre de 2014.

APROBACIÓN: 25 de septiembre de 2015.

* Departamento de Filosofía. Instituto Superior de Estudios Eclesiásticos. 
CITAM Derechos Reservados.

La reproducción total o parcial de este artículo se podrá hacer si el ITAM otorga la autorización previamente por escrito. 


\section{LA SIGNIFICACIÓN ONTOLÓGICA DE LA PRIMORDIALIDAD DE LA SUSTANCIA EN ARISTÓTELES}

\section{Introducción}

\section{Elobjetivo del presente estudio es acla-} rar qué significa la primordialidad de la sustancia en el tratamiento sobre la oucía que desarrolla Aristóteles en el libro Z de Metafisica, su tratado de filosofía primera. De manera particular nos interesa poner a la vista qué sentido tiene la primordialidad de la sustancia según el tiempo. ${ }^{1}$ Para llevarlo a cabo, comenzaremos por exponer de manera sucinta qué lugar ocupa la pregunta por la sustancia en la investigación filosófica del Estagirita, es decir, aclararemos qué relación guardan la pregunta por el ente en cuanto tal y la pregunta por la sustancia. Una vez expuesto lo anterior, nos adentraremos en el libro Z de la Metafísica, lugar en el que nuestro autor desarrolla parte de su concepción acerca de la sustancia, a saber, la que tiene como meta exponerla como sujeto, como forma, como universal y como género. Cuál

${ }^{1}$ Un tratamiento exhaustivo y destacado del concepto de primordialidad, o prioridad $-\mathrm{y}$ que es de relevancia en nuestro trabajo-, lo encontramos en el capítulo primero de los estudios aristotélicos de Alejandro Vigo. Ahí, Vigo se encarga de explorar las conexiones de los significados de prioridad a partir del capítulo XII de Categorías y del libro $\Delta$, capítulo 11 de la Metafísica; véase A. Vigo, Estudios aristotélicos, 2006, Navarra, Eunsa, pp. 23-54. Más cercano a nuestro tema, y con una finalidad eminentemente expositiva de la dificultad en torno a la primordialidad, se encuentra el estudio de Christof Rapp, titulado "Substanz als vorrangig Seindes (Z1)" recogido en Metaphisik. Die Substanzbücher (Z, H, $\Theta)$; 1996, Tubinga, Akademie Verlag, pp. 27-40. 
de estas sea la determinación de la sustancia ha de responder necesariamente a un criterio. En el capítulo segundo de dicho libro, Aristóteles señala los tres aspectos en los que la sustancia ha de ser considerada como el ente primordial ( $\tau$ ò $\pi \rho \tilde{\tau} \tau o v)$ : según el conocimiento (primordialidad epistemológica), según la definición (primordialidad lógica) y según el tiempo (primordialidad física). ${ }^{2}$ Estos tres sentidos, a nuestro parecer, sirven a lo largo del libro $\mathrm{Z}$ como criterios para delimitar qué debe ser entendido propiamente como sustancia, esto es, fungen como delimitación metódica para poner a la vista los rasgos propios de la sustancia. Pero no solo esto, sino que también, en tanto que se trata de la primordialidad de la sustancia en sentido ontológico, el examen de los tres modos de primordialidad deja ver el ordenamiento que hay en lo que se dice que es dentro de las pretensiones de la ciencia primera. A nuestro parecer, la primordialidad en cuanto al tiempo cumple una función fundamental dentro de los criterios señalados. La primordialidad ontológica da lugar, pues, a una primordialidad en el orden tanto lógico, como en el orden del conocimiento. Ya la noción de $\tau$ ò $\pi \rho \tilde{\omega} \tau o v$ sugiere que en lo ente en su totalidad existe una ordenación, y por lo mismo cabe indagar qué y cómo es aquello que funda dicho orden. Por ello, consideramos necesario poner en claro qué es lo que se entiende por primordialidad de la sustancia.

Por lo que respecta a los dos primeros sentidos de lo primordial - en cuanto al conocimiento y en cuanto a la definición-, Aristóteles sí ofrece indicaciones que ayudan a entender los desarrollos de los problemas que se plantean en los capítulos posteriores a Z 2. En cambio, respecto de la primordialidad en cuanto al tiempo, el Estagirita no dice nada claro. Al terminar de enunciar los sentidos de la primordialidad, tiene una expresión que parece apuntar más a la importancia de la cuestión en torno a la sustancia, que a destacar el sentido de la primordialidad temporal. Dice así: "Y en efecto, lo que antiguamente y ahora y siempre se ha buscado y siempre ha sido objeto de duda:

${ }^{2}$ Cfr. Metaph. Z 1028a 31-33. Sigo la traducción de Valentín García Yebra, Metafísica, 1982, Madrid, Gredos. 
¿qué es el Ente? equivale a ¿qué es la Substancia?”. ${ }^{3}$ A partir de esto, solo cabe decir que la pregunta acerca de la sustancia es la pregunta fundamental que atraviesa a toda la historia de la filosofía, pero con ello no se plantea aún el problema de la relación entre la sustancia y el tiempo. Debido a lo anterior, creemos que es necesario pensar cuál es la relación que sostiene la sustancia con el tiempo, pues solo de este modo podremos arrojar luz sobre la dificultad inherente a aquella relación. Cuando hablamos de relación, no se debe pensar como si sustancia y tiempo fuesen dos realidades distintas, pues ello supondría un dualismo que Aristóteles parece no sostener; antes bien, la relación está pensada aquí en cómo se corresponden la sustancia y el orden temporal, en cómo aquella es lo primero en el orden del tiempo y cómo este necesita aquella. Nuestra tesis acerca de cómo se puede aclarar la primordialidad en cuanto al tiempo consiste en que el problema de la relación entre sustancia y tiempo se encuentra desarrollado a partir de la interrogante acerca de qué es el movimiento y cómo se da. En todo caso, ha de

${ }^{3}$ Arist. Metaph. Z 1028b 2-4. Ciertamente Aristóteles señala, inmediatamente después de haber enunciado los tres sentidos en los que se dice que la sustancia es lo primero o primordial,

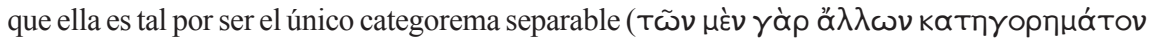

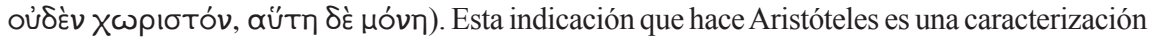
propia de la sustancia que, en rigor, vale para los tres sentidos en que ella es lo primordial. O dicho de otro modo, la separabilidad de la sustancia es una consecuencia de que ella sea, a una, lo primordial en cuanto al tiempo, en cuanto al enunciado y en cuanto al conocimiento. Que la sustancia sea separable significa que solo ella tiene en grado máximo existencia por sí ( $\kappa \propto \theta^{\prime} \propto \cup \dot{T}$ ó), esto es, no depende de otro modo de ser del ente ni para ser lo que es ni para existir, a diferencia de los demás categoremas accidentales ( $\sigma \cup \mu \beta \varepsilon \beta \eta \kappa o ́ \varsigma)$ que sí necesitan de algo, de la sustancia, para existir. Los llamados categoremas accidentales no son separables puesto que, aun cuando cada uno, en cuanto se da, representa un momento de ser que posee cierta consistencia ontológica, sin embargo están necesitados de algo más para darse. Esto quiere decir que siempre se dan (ن́rTópXєાv) en otro, se dan en algo que ya existe, o se dan como aquello sobre lo que versa la predicación de algo. Así, los accidentes suponen un principio que hace posible su existir, sin que por ello el existir de lo supuesto y el existir de lo accidental sean esencialmente lo mismo. Por tanto, la idea de lo separable apunta a que solo la sustancia posee autosuficiencia ontológica plena, la cual puede ser comprendida en tanto se aclare el triple orden de primordialidad de la sustancia. Sin detrimento de esto, la existencia de lo accidental también se da, y de algún modo como algo por sí puesto que hablamos de ello, pero no como un ser separable, sino como una manera de ser que se da en otro. En ello radica el rango y modo de su cognoscibilidad. Y así, hablando de los accidentes, dice Aristóteles: "también conocemos cada una de estas mismas cosas cuando sabemos qué es la cantidad o la cualidad" (Metaph. Z 1028a 1-2). Véase también el texto de Rapp, op. cit., pp. 34-35. 
tenerse presente la tesis aristotélica del que el tiempo es, o una afección el movimiento, o bien algo relativo a este. Por ello, nos limitaremos a desarrollar las ideas que Aristóteles expone a partir de este tema. Dado que el tiempo acompaña en todo caso al movimiento, es necesario mostrar a aquel a partir de este. Para ello seguiremos los tratamientos que da el Estagirita al movimiento en Acerca de la generación y la corrupción y en la Física. Puesto que nos atenemos solo a una parte del problema de la primordialidad de la sustancia, no pretendemos dar por agotada la investigación del asunto. En todo caso, lo que nos interesará, a modo de conclusión, será señalar los problemas que quedan abiertos en torno a la primordialidad de la sustancia a partir de esta interpretación que proponemos.

\section{La cuestionabilidad de lo ente y la necesidad de la pregunta por la sustancia}

La pregunta conductora de la Metafisica es la que interroga por el ente en cuanto tal y lo que le corresponde de suyo. ${ }^{4}$ Con ello el Estagirita solo hace manifiesta la dirección a partir de la que se encamina la investigación fundamental de la filosofía, la ciencia primera. Y decimos solo porque la filosofía primera no es algo que esté ahí ya a disposición de nosotros por el simple hecho de señalar el objeto del que se ocupa, sino que en todo caso debe desplegar su investigación y debe tratar de consolidarse como ciencia. Se trata de una ciencia buscada. La realización de la ciencia del ente en tanto que ente es lo buscado en la investigación que se dirige hacia el ente en cuanto tal y en lo que le corresponde de suyo, es decir, la meta de la filosofía está en que sea no solo una investigación acerca del ente en cuanto tal, sino también en que se consolide como un saber fundamental de la totalidad de lo ente. En este sentido, el asunto radica en qué medida, dentro del investigar mismo, el ente en cuanto tal se hace cognoscible no solo en su inmediatez, sino también en sus rasgos fundamentales. Pero si se trata de un dejar

${ }^{4}$ Cfr. Metaph. Г 1003a 21-22. 
hacerse cognoscible del ente, entonces en ello se supone una inmediata duplicidad que se da en nuestra relación con lo ente: por un lado, es necesario que lo ente nos sea ya accesible, y por otro, que desde esto accesible se nos abra el camino hacia aquello que no se deja ver a simple vista, pero que es objeto de cuestionamiento. ¿En qué radica esa forma de inmediatez que abre el camino para lo cuestionable? En que lo que vemos que es, y que es aquello de lo que hablamos inexcusablemente, está presente de diversas maneras; sin embargo, esa diversidad no está presente en dispersión, sino que se mueve siempre en un único ámbito: que es. Cómo sea posible que se dé esa diversidad en un único ámbito es lo digno de ser interrogado. Cómo es que el universo está constituido de tal modo que sea un todo dispuesto en una totalidad de partes, las cuales convergen en el hecho de ser, se erige como el hecho asombroso para la posibilidad de la ciencia primera.

Aristóteles reconoce en el libro $\Gamma$ que: "el ente se dice en varios sentidos, aunque en orden a una sola cierta naturaleza única". ${ }^{5} \mathrm{La}$ dificultad a la que se enfrenta la filosofía primera - que bien podría llamarse ontología en el sentido de tener una visión en conjunto de lo ente- consiste en distinguir los diversos sentidos en los que puede hablarse de ente, y alcanzar a comprender qué significa que esos diversos sentidos de decirse el ente estén referidos a una sola cosa

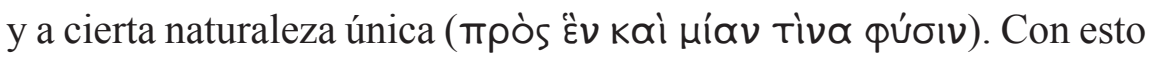
queda perfilado el método que guía las investigaciones de Aristóteles, el cual consiste en un discernimiento de los modos de decirse el ente siempre en vista a esa naturaleza única de la que dependen los diversos modos de decirse el ente. Aquí no debe perderse de vista aquello que señala el Estagirita acerca de la amplitud real, y no meramente significativa, que comparten el término ente y el término uno: “cuantas sean las especies del Uno, tantas serán las del ente, acerca de las cuales corresponde a una ciencia genéricamente una contemplar la quididad". ${ }^{6}$ Por ello, Aristóteles no busca solamente discernir los diversos significados que tiene la palabra ente, sino que también busca

${ }^{5}$ Metaph. Г 1033a 33-34.

${ }^{6}$ Metaph. $\Gamma$ 1033b 33-35. 
conocer lo esencial del principio que posibilita los diversos modos de darse el ente, no como algo meramente accidental, sino como esa diversidad que se corresponde con el propio ser del principio. ${ }^{7}$ En esto radica la más grave dificultad de la ciencia primera: no se trata únicamente del conocimiento de los principios del ente, sino de todo lo que de ahí se sigue. En relación con la ciencia del ente en cuanto tal, empleando esta metodología que acabamos de señalar, dice el Estagirita que: "la ciencia trata propiamente de lo primero, y de aquello de lo que dependen las demás cosas y por lo cual se dicen. Por consiguiente, si esto es la substancia, de las substancias tendrá que conocer los principios y las causas el filósofo". ${ }^{8}$ Como podemos ver, para el Estagirita saber qué es lo que da unidad al método es también un problema, pues en estos pasajes del libro $\Gamma$ no parte de la evidencia de qué sea aquello en lo que consiste el objeto propio de la ciencia que se busca, ni qué sea esa única naturaleza que da unidad al método de la filosofía primera. De momento, lo mentado con la palabra sustancia es apenas aquello con lo que se pretende designar un punto de mira, pero no es una evidencia, pues aún queda por investigar los principios y causas de la sustancia. Así, se trata todavía de una investigación no solo de la sustancia, sino también de lo que le corresponde de suyo (k đì Tò

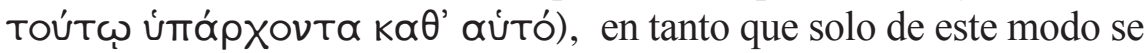
va aclarando el modo de ser de la ouбía. Por ello, es necesario que el filósofo teorice de algún modo acerca de todas las cosas, esto es, de todo aquello que es, teniendo a la vista que lo buscado es cómo existe aquello que deja estar en unidad a la diversidad de lo que existe. Todo esto quiere decir, en palabras de Aristóteles, lo siguiente: "pero todo ente se dice en orden a un solo principio. Unos, en efecto, se dicen entes porque son substancias; otros porque son afecciones de las substancias; otros, porque son camino a la substancia, o corrupción o privación o cualidades de la substancia o las cosas dichas en orden a

${ }^{7}$ Aquí queremos insistir en que el modo de ser de lo accidental, si bien es un ser dependiente o no separable plenamente, también es algo que, de algún modo, posee esencia y es algo por sí. De lo contrario no solo no tendría cognoscibilidad ni podríamos hablar de ello, sino que tampoco tendría existencia. Lo que diferencia al accidente es el modo de su esencia y su manera de ser por sí, pero en principio es; y si bien su existencia puede ser todo lo azarosa e inexplicable que se quiera, no por ello es gratuita o excedente en el reino del ente.

${ }^{8}$ Metaph. Г 1003b 16-19. 
la substancia, o porque son negaciones de alguna de estas cosas o de la substancia. Por eso también decimos que el No-ente es No-ente". 9 De este modo, aun cuando la investigación de lo ente es tan extensa como modos de ser se dan, también queda señalado que parece haber un modo de ser del que todo modo de hablar y del que todo modo de ser del ente dependen, es decir, la sustancia, razón por la cual es preciso indagar qué significa su naturaleza primordial. La dificultad metodológica de la ontología radica, entonces, en no perder de vista aquello con vistas a lo cual se da y se dice lo ente. ${ }^{10}$

Antes de dar un paso más, es oportuno detenernos en algo que no es de poca importancia para nuestra investigación. ¿Por qué Aristóteles ha señalado como problema fundamental de la filosofía primera el hecho de que el ente se dé y se diga de diversos modos? ¿Con quién dialoga nuestro autor al plantear la cuestión de esta forma? Con el planteamiento ya señalado, quiere el Estagirita poner a la vista que la antigua doctrina parmenídea que afirmaba la univocidad de lo que el ente es en sí, entraña una dificultad que ha sido dejada de lado. Dicha dificultad consiste en que, atendiendo a cómo de hecho se dan las cosas, si bien todo aquello acerca de lo que se puede decir o pensar es algo de algún u otro modo, precisamente por existir diversos modos de darse y de decirse el ente, se hace necesario pensar qué es lo que da unidad a aquellos diversos modos de ser del ente, a la vez que esa unidad es

${ }^{9}$ Metaph. Г 1033b 5-10.

${ }^{10}$ Con lo dicho hasta aquí se dejan ver los rasgos esenciales de la ontología fundamental como ciencia universal. Para Aristóteles no resulta absurdo pensar que la filosofía primera no tenga que ocuparse a cabalidad de todo lo que existe. Cuando se dice que se trata de una ciencia de lo ente en cuanto tal, si bien con ello se dirige la mirada a lo que de común tiene todo lo que existe, sin embargo a la vez con ello queda fuera del círculo de problemas de la ontología todo aquello que es el ente en cuanto determinado de esta o aquella manera. Se trata, pues, de una ciencia que tiene bien delimitado su marco de despliegue, y es en dicho marco que, con apego a los asuntos mismos, se plantean los problemas a los que se enfrenta. Pero con esto tampoco se quiere decir que los avances de las ciencias particulares sean del todo irrelevantes para la ciencia primera, en tanto que aquellas, aun dentro de su marco particular, están referidas en primera y en última instancia a algo que es. Sería un acto de omisión culposa el que la ciencia primera no considerara los avances de estas disciplinas; se trataría de esa desatención que traicionaría la intención filosófica de teorizar acerca de todo en la medida de lo posible. Antes bien, en la medida en que se atiende al espíritu del filosofar, queda abierto el problema de cómo se puede transitar del conocimiento de las ciencias particulares al círculo de cuestiones propias de la ciencia del ente en cuanto ente. 
lo que permite que se den las múltiples modalidades del ente. ${ }^{11}$ Se trata, pues, del problema acerca de la unidad y multiplicidad que constituye a la totalidad del ente, problema que también desarrolla Aristóteles a lo largo del libro $\Lambda$ de la Metafísica, pero ya teniendo como punto de partida a la sustancia. En este sentido, leemos en el libro XII lo siguiente: "En efecto, si el Universo es como un todo, la sustancia es su primera

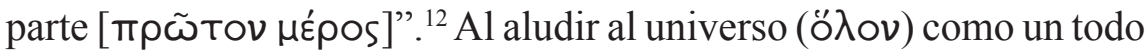
( $\pi \tilde{\alpha} \nu)$, el Estagirita quiere hacer énfasis en la no absoluta identidad de todo lo que es, pero también quiere destacar que esa no identidad no implica que existan muchas realidades, sino una sola. No se trata de una superación de alguna forma de dualismo, sino de pensar la conge-

${ }^{11}$ Para Aristóteles, la desatención a este problema se debe a que Parménides se atuvo primordialmente a la correspondencia lógica entre el pensar y el ser, dejando de lado que aun el pensar mismo está en íntima conexión con los fenómenos, los cuales son una prueba suficiente para mostrar que lo que es, el ente, no se da siempre del mismo modo, y lo que es más, que los diversos modos de darse el ente parecen guardar entre sí una relación muy bien definida. Esto no significa que Aristóteles descalifique por completo la doctrina parmenídea; más bien, Aristóteles considera que ha sido Parménides quien ha alcanzado a ver la unidad de la totalidad de lo ente, pero ha dejado de lado el problema de la relación que sostienen la unidad y la pluralidad en el ámbito de los fenómenos, y esto en razón de que la unidad que Parménides ha descubierto se sostiene en buena medida solo dentro del ámbito lógico. En efecto, todo lo que puede ser pensado y dicho, aun cuando esté ausente, tiene como carácter fundamental el es. El pensar y el decir son así una vía para ir más allá de la inmediata presencia evanescente que contemplamos con los sentidos, pues a ellos se impone la insuperable presencia del Tó દőv. El mismo Aristóteles llega reconocer algo semejante, pero le imprime un giro al querer poner atención en las diferencias: "puesto que, de los predicados, unos significan quididad, otros cualidad, otros cantidad, otros relación, otros acción o pasión, otros lugar y otros tiempo, el ser significa lo mismo que cada uno de estos" (Metaph. $\Delta$ 1017a 24-27). Tenemos, pues, que todo lo que se puede decir, y sobre todo pensar, es. Pero - y aquí está el peso del discernimiento de Aristóteles - a lo que se apunta en concreto con cada una de esas predicaciones no es idéntico en todos los casos. No es este el lugar para discutir con detalle si la interpretación que hace el Estagirita del pensamiento de Parménides es acertada o no; se podría aducir, quizás, que se trata de una discusión con Zenón de Elea. Pero lo que sí resulta significativo es que desde la perspectiva de Aristóteles, resulta esencial prestar atención a las diferentes maneras de ser que se perciben inmediatamente, pues son dichas diferencias las que, por decirlo así, condicionan el modo de pensar y de hablar de las cosas. La pluralidad de lo ente no es asunto de una opinión humana, sino la realidad de hecho. De este modo, Aristóteles quiere mostrar cómo es que la unidad de la totalidad de lo ente no se limita al espacio lógico, sino que funda y atraviesa a la realidad entera, y lo hace de tal modo que dicha unidad no existe en detrimento de la pluralidad, sino que precisamente es aquello que permite que la pluralidad exista tal y como es, es decir, como el ensamble de una totalidad cuya parte primordial reside en un peculiar modo de ser del ente, en la sustancia.

${ }^{12}$ Metaph. $\Lambda$ 1069a 19-20. 
neridad de lo uno y lo múltiple desde esa primera parte del todo, la cual está significada por el término 'sustancia'. Así, insistimos, es preciso indagar, hasta donde sea posible, la manera en que la sustancia existe como la primera parte de la totalidad del universo - entendiendo aquí que 'primera parte' significa aquello que da cohesión y unidad a la constitución de la totalidad del universo, a la vez que es aquello que da lugar a la multiplicidad de lo ente- - Con esto, ha quedado reforzada nues-

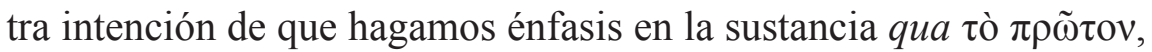
pues con ello se busca no solo lo primero —o dicho de modo más preciso, lo primordial - en el orden del conocimiento, del enunciado y del tiempo, sino en el orden del universo entero, así como también qué significa esto primordial en relación con los otros modos de ser del ente que bien puede ser entendida en general como primordialidad cosmológica.

\section{Consideraciones generales sobre la primordialidad ontológica de la sustancia frente a los accidentes dentro del orden ontológico-categorial}

Con lo que acabamos de señalar ya hemos anticipado que para Aristóteles, si bien existen múltiples modos de ser y de hablar del ente, el sentido primario del es corresponde a la sustancia. La sustancia es la naturaleza propia e íntima de las cosas, esto es, el principio a partir del cual están determinadas a ser lo que son. Pero no solo esto, sino que en el orden del decir — el cual está enmarcado en el orden categorial—al aludir al qué-es, con ello se apunta hacia aquella naturaleza propia e íntima. La razón que da Aristóteles en un primer momento a favor de la primordialidad de la sustancia consiste en que, cuando decimos que algo es de tal o cual modo - lo cual solo es posible porque ese algo se acusa, se categoriza de tal o cual modo - con ello no nos estamos refiriendo a lo que la cosa propiamente es, sino que nos limitamos a señalar alguna determinación suya que, sí nos dice algo de la cosa, pero que no se ajusta con el ser propio de la cosa. Por el contrario, cuando nos referimos a la cosa en virtud de su qué-es (Tí ÉoTı) en la que se apunta a 
la sustancia, sí nos estamos refiriendo al ser propio y constitutivo de dicha cosa. Así, cuando decimos que algo es de tal tamaño, solo nos estamos refiriendo a una determinación parcial de la cosa, y con ello dejamos de lado lo que la cosa propiamente es, o bien no atendemos a otras determinaciones parciales de la cosa. Pero cuando decimos que tal cosa es un hombre o un dios, y nos damos a la tarea de definir qué-es el ser del hombre o el ser del dios - lo cual solo es posible porque ambas cosas se acusan según esa concreta manera de ser- nos estamos refiriendo al principio constitutivo que funge como la determinación fundamental y concreta del ser de la cosa. En el orden categorial, los accidentes son ciertamente algo, es decir, acusan una manera o momento de lo ente, pero esto que se acusa es precisamente accidental por estar necesitado de la sustancia, y por corresponderse con ella. En relación con esto, en los Analíticos segundos podemos leer lo siguiente: "[los predicados] que significan la entidad [ouбía] significan que aquello acerca de lo cual se predican es precisamente tal cosa o un tipo de ella, en cambio, todos los que no significan la entidad, sino que se dicen acerca de un sujeto distinto, que no es, ni lo que precisamente es aquel [predicado], ni algún tipo de este, son accidentes, v.g.: blanco acerca de hombre". ${ }^{13}$

El hecho de que las afecciones de las sustancias, los accidentes, pertenezcan siempre y en todo punto a la sustancia se hace visible a partir de que los accidentes del ente no tienen un principio de existencia propia, ni pueden separarse de la sustancia. De este modo, dice Aristóteles que toda determinación parcial de la cosa, sea cantidad, cualidad, afección o cualquier otra determinación de este tipo —que como sabemos, se llaman accidentes - solo llega a ser (úrrópXદıv) en tanto que exista como algo que pertenece a una sustancia; los accidentes suponen un principio que los posibilita, a la vez que ellos se dan en el orden que dicho principio, la sustancia, funda. Se trata aquí del empleo de la distinción ontológica entre lo ente por sí y lo accidental que enuncia el Estagirita en el libro $\Delta .{ }^{14}$ Por ejemplo, para que llegue

${ }^{13}$ Arist. An. Post. 83a 24. Sigo la traducción de Miguel Candel Sanmartín, Tratados de lógica II, 1988, Madrid, Gredos.

${ }^{14}$ Una destacada aclaración de la noción de prioridad ontológica puede verse en: A. Vigo, "Prioridad lógica y prioridad ontológica en la doctrina aristotélica de la sustancia", Estudios Aristotélicos, 2006, Navarra, Eunsa, pp. 55-85. 
a existir el estar-sentado - como un momento en el orden categorial de lo ente al que llamamos "posición"- es necesario que haya algo concreto, determinado y por sí, que esté sentado; o dicho de otro modo, el estar-sentado no tiene existencia independiente ni puede estar separado del ente que, sirviendo de sustrato al accidente, en primer lugar es algo por sí, y por ello, puede estar sentado. A partir de esto se hace visible que los llamados accidentes dependen ontológicamente de la sustancia, es decir, están necesitados de la sustancia para poder llegar a existir; aun cuando el momento del ser que se acusa categorialmente en ellos es algo determinado — razón por la cual los predicados tienen significación-, tal acusarse solo es posible porque de fondo hay un sujeto de predicación que hace posible el sentido en la predicación. Este estar-necesitado que corresponde al modo de ser de los accidentes indica, pues, que lo primordial en la constitución ontológica de las cosas reside en la sustancia, que es lo por sí, y también hace evidente que todo hablar del ente, a partir de categorías o predicamentos distintos a la de la quididad, apunta en última instancia al ente que es sujeto último de toda predicación, es decir, a la sustancia.

\section{Sobre la primordialidad de la sustancia en cuanto al enunciado y en cuanto al conocimiento}

En razón de que la sustancia es aquello que sirve necesariamente de soporte a toda posible afección, a todo accidente, se acredita como el modo primordial de ser del ente. Ciertamente se nos ha hecho visible que la sustancia es lo primordial en virtud de que todos los otros modos de ser del ente dependen de ella, pero con ello no hemos dicho plenamente qué significa la primordialidad de la sustancia. Y no lo hemos hecho porque Aristóteles reconoce, una vez más en Metafísica Z 1028a 31, que primordial se dice en varios sentidos, lo cual no va en detrimento de que todos estos sentidos se refieren al modo de ser propio de la sustancia, y no a otra cosa. La dificultad acerca de la primordialidad de la sustancia no apunta a si con aquella se hace alusión a un modo de ser distinto de la sustancia, sino que consiste en 
alcanzar claridad y precisión acerca de cada uno de los sentidos en que se nombra la primordialidad de la sustancia. Esto quiere decir que si bien la sustancia es esa única naturaleza que puede ser llamada primordial, los diversos sentidos propios de la primordialidad destacan distintos órdenes en los que se da dicha primordialidad, órdenes con arreglo a los cuales se determina qué de lo que existe puede ser denominado como sustancia. Y por ello la pregunta por la primordialidad requiere un planteamiento preciso en el que se destaque a qué se refiere propiamente cada uno de sus sentidos. ¿Cuáles son los múltiples sentidos en los que se dice o se da la primordialidad? Aristóteles destaca tres: en cuanto al enunciado ( $\lambda$ ó $\gamma$ os), en cuanto al conocimiento ( $\gamma \nu \tilde{\omega} \sigma ı)$ y en cuanto al tiempo ( $x$ póvos). Por tanto, a continuación trataremos de plantear correctamente la pregunta por cada uno de estos sentidos, a fin de que con ello nos quede claro en qué consisten, y de modo señalado, qué significa la primordialidad de la sustancia en cuanto al tiempo.

¿Qué significa la primordialidad en cuanto al enunciado? Para poder saber esto es necesario que indaguemos antes cuál es la forma propia del enunciado ( $\lambda$ óyos). En todo enunciado tenemos que se dice algo de algo, en el que el primer algo es la predicación de un determinado estado del segundo algo que funge como sujeto de predicación. Sobre esta base, ahora preguntamos: ¿sería posible predicar algo sin que hubiese un sujeto al que esté referido dicho predicado? Más arriba hemos señalado que el estar-sentado - que indica una determinada posición - no puede existir por sí mismo, esto es, no puede darse sin que haya un ente que lleve a cabo la acción de sentarse o que se encuentre ya en dicha posición. Y del mismo modo que esto, el estar-sentado, no puede darse sin algo que esté en dicha posición, tampoco podemos decir que el estar-sentado exista con independencia de aquello de lo que estamos diciendo que se encuentra en tal posición. Aunque digamos simplemente 'está sentado', o intentemos decir abstractamente 'estarsentado', con estos enunciados estamos, implícitamente, refiriéndonos a algo que sirve como sujeto de predicación, es decir, estamos diciendo que un algo es lo que lleva a cabo la acción de estar sentado o que se encuentra en dicha posición, esto es, un algo que está sentado. Por ello dice 
Aristóteles que la primordialidad de la sustancia en cuanto al enunciado consiste en que "en el enunciado de cada cosa entrará necesariamente el de la Substancia". ${ }^{15}$

Esto no quiere decir que cuando proferimos el enunciado 'algo está sentado’ con ello estemos diciendo qué es tal cosa que está sentada, sino que nos limitamos a poner de manifiesto un determinado estado de cosas al que nos referimos, esto es, nos limitamos a decir que tal cosa está en tal posición, dejando con ello de lado el qué propio o sustancial de la cosa. No obstante, también pudiera ser el caso de que en nuestro enunciado estemos diciendo qué es tal cosa, que estemos poniendo de manifiesto la definición (ópıouós) propia de la cosa.

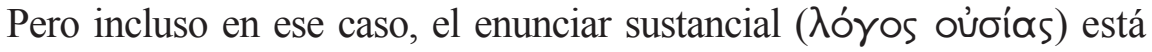
referido a un sujeto concreto y determinado que sirva de referente último para la definición; lo que se expresa en la definición no es un momento meramente lógico, sino que lo enunciado en la definición es de la cosa, de su qué-es. ${ }^{16}$ Así, lo que Aristóteles quiere decir con la primordialidad de la sustancia en cuanto al enunciado se refiere a que en toda predicación se apunta, ya sea de un modo esencial o no, a la sustancia que sirve de término último de la predicación. Ésta es la razón por la cual en todo enunciado se nombra, de modo explícito o no, a la sustancia respecto de la cual se enuncia algo. Y lo que es más, solo la

${ }^{15}$ Metaph. Z 1028a 35.

${ }^{16}$ Aquí es preciso aclarar cómo es que definición solo puede haber de la sustancia, o bien, solo se define un ente en tanto se le considera en su qué. En contra de lo que usualmente se cree acerca de la definición - la cual es tomada como definición de un concepto y no de una cosa-, el ópıouós se refiere ante todo al qué-es de lo ente. Acerca de esto, Nicolai Hartmann da una explicación bastante razonable: "Lo que el ópı

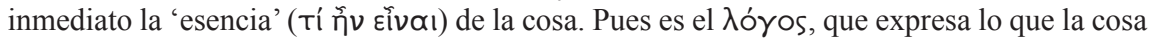
propiamente es. Si solo se dijera lo que es el 'concepto' de la cosa, resultaría, precisamente según las presuposiciones de esta interpretación, un contrasentido: primero se entiende el $\lambda$ ó como concepto, y luego se hace al concepto objeto de la definición, la que a su vez, no obs-

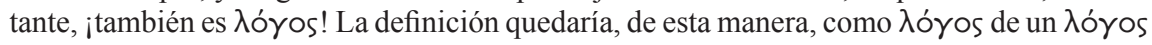
- lo que evidentemente no tiene ya nada de común con los procesos del pensar aristotélico"; (Aristóteles y el problema del concepto, 1964, México, UNAM, trad. de Bernabé Navarro, p. 18). Así, la primordialidad de la sustancia en cuanto a la enunciación no es meramente lógica - pues esto supondría que lo definido es un concepto-, sino onto-lógica. Lo definido y de lo cual puede resultar algo así como un concepto es la esencia de lo ente - que se da primordialmente en la sustancia, y solo secundariamente en las otras categorías - la cual queda expresada

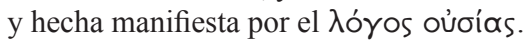


sustancia es algo definible por sí, es decir, solo de ella existe una definición en la que la cosa es definida por sí misma a partir de su naturaleza propia y no en virtud de otra. Esto se hace evidente más aún si tomamos en cuenta que cuando queremos decir qué es una cualidad o cualquiera de las otras categorías, o cuando queremos decir qué es la corrupción o generación, en ambos casos nombramos como sujeto de predicación a algo que sirve de sustrato; por ejemplo, cuando decimos qué es el ser blanco — en caso de que existiera definición de este modo de ser ente- nombramos necesariamente aquello en lo que se da dicho color - a saber, un cuerpo - y cuál es la cualidad de dicho color. Pero no podemos decir cuál es la cualidad del color blanco sin que antes mentemos, de forma explícita o no, una sustancia que le sirve de sustrato. En cambio, solo las sustancias pueden ser definidas por sí mismas sin hacer alusión a otra cosa y con independencia de los accidentes que les afectan. Por ejemplo, para definir qué es este hombre no es necesario aludir a los accidentes que le sobrevienen, como pueden ser la situación, la relación o el número, sino que basta con mirar al hombre mismo en lo que tiene de propio y constitutivo, a saber, su alma racional. ${ }^{17}$

Por lo que respecta a la primordialidad de la sustancia en cuanto al conocimiento, el planteamiento correcto de la pregunta consiste en que interroguemos cuándo y cómo es que conocemos a una cosa en su fundamento. Por principio, y en correspondencia con las primeras líneas de la Metafísica, Aristóteles no niega que los accidentes de las cosas nos den cierto conocimiento de ellas; por ejemplo, el saber que el perro posee cuatro patas o que la mesa es de tal tamaño y que tiene tal color nos da información de la cosa. Sin embargo, dicha información

${ }^{17} \mathrm{Si}$ aquí aludimos a la forma como aquello que es definido, y que por ello, es la que propiamente puede ser llamada sustancia, se debe a que la materia es, por sí misma, indefinible. En Metafísica Z 1037a 24-30 podemos leer lo siguiente: "las partes materiales no están incluidas en el enunciado de la substancia, pues no son parte de la substancia, sino del todo concreto, del cual en cierto modo hay y no hay enunciado; junto con la materia no lo hay (pues es algo indeterminado) pero lo hay con referencia a la substancia primera [...] la substancia, en efecto, es la especie inmanente, de cuya unión con la materia procede lo que llamamos sustancia concreta". Con base en esto, se nos ofrece una primera indicación de que la sustancia en sentido primordial, según la definición, es la forma y no la materia, pues solo la forma es separable, abstraíble, y por tanto, definible por sí. 
no nos da razón del ser de la cosa, no apunta a su fundamento o principios. Para Aristóteles, el conocimiento se da en mayor grado, según ha dicho en A 982b 2, cuando conocemos los principios y las causas de las cosas. Y dentro de los principios y causas, que son el fundamento de la existencia de las cosas, está precisamente la causa formal: la sustancia y la esencia. ${ }^{18}$ De este modo, si bien hay cierto conocimiento a través de la percepción de los accidentes — que son lo más accesible para nosotros-, este conocimiento no es el más alto porque no nos da el fundamento de las cosas, y lo que es aún más, presupone y depende del fundamento del ser de las cosas. Indudablemente, esto no quiere decir que el conocimiento de los accidentes - que se da principalmente a través de la sensibilidad - sea algo prescindible, pues solo podemos llegar a fijar nuestra mirada investigadora en las sustancias de las cosas en tanto que estas se nos anuncian únicamente a través de sus determinaciones accidentales. Sin embargo, el que busca un auténtico saber de la totalidad del ente es necesario que vaya más allá, que radicalice en el conocimiento proveniente de los accidentes y de la sensibilidad para así alcanzar el fundamento de las cosas, el cual descansa primordialmente en la sustancia. La primordialidad de la sustancia en cuanto al conocimiento consiste, por tanto, en que, en atención a un saber fundamental del ente, solo llegamos a saber algo de los entes en lo que tienen de propio cuando conocemos ese modo de ser primordial que es la sustancia. Si bien los accidentes son lo primero que conocemos - porque ellos son lo inmediatamente accesible para el conocimiento-, en

\footnotetext{
${ }^{18}$ Aquí debemos destacar que para las cosas físicas — es decir, las que existen como compuestas y sujetas al movimiento- no solo la sustancia y la esencia son principios constitutivos de su existencia, sino que también lo es su materia. Sin embargo, la materia es un principio de existencia que por sí mismo es indeterminado - es decir, la materia es un principio de las cosas que consiste en la potencia de ser o no ser-y por ello, es incognoscible, tal como Aristóteles afirma en Metafísica Z 1036a 8-9: "Y la materia en cuanto tal es incog-

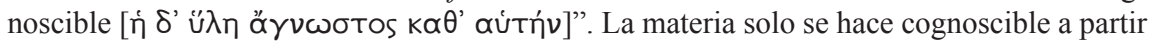
de que ella está necesariamente unida, en el ámbito físico, a una sustancia determinada, esto es, a una forma que funge como el principio que la determina. Por ello, en el orden del conocimiento, la materia no es sustancia primordial, sino solo la forma, es decir, la sustancia y la esencia. Con base en esta indicación, podríamos deducir que si lo que puede ser llamado sustancia solo corresponde a aquello que es primordial en todos los sentidos en que esto puede ser dicho, entonces la materia, al no ser cognoscible por sí, no es sustancia primordial, pero sí lo es la forma, pues ella es definible y cognoscible por sí.
} 
rigor, ellos sirven como camino para un conocimiento de la sustancia, que es lo primero cognoscible en sí. Esto se debe a que los accidentes apuntan a algo que necesariamente les subyace, y de este modo, a que ellos solo son cognoscibles en tanto que hay una sustancia que los soporta, es decir, que hace posible su cognoscibilidad.

\section{La sustancia y su preexistencia en la generación y la corrupción}

Ya hemos dicho al comienzo de nuestro estudio que la primordialidad de la sustancia en cuanto al tiempo no parece estar señalada por Aristóteles a partir de que ha sido el problema al que se ha enfrentado la filosofía a lo largo de su historia. Sobre la base de esta afirmación, hemos dicho que con ello no se plantea propiamente el problema de la relación de la sustancia con el tiempo. Con esto no queremos decir que haya una omisión por parte de Aristóteles respecto a la dificultad ya señalada; a nuestro parecer, el problema propio de la relación acerca de la sustancia y el tiempo está desarrollado a partir de la interrogante por la generación, cuestión que se aborda, desde la perspectiva de la primordialidad, en los capítulos 7-9 del libro Z. En estos capítulos se deja ver que la primordialidad de la sustancia en cuanto al tiempo reside en que preexiste al propio movimiento de generación y corrupción de los entes. ${ }^{19}$ Esta preexistencia, insistimos, no debe ser entendida como si la sustancia estuviese separada ontológicamente de los entes físicos, sino que más bien se refiere a aquello que da soporte, unidad y continuidad a los distintos tipos de movimiento de las cosas en el ámbito físico. ${ }^{20} \mathrm{En}$ efecto, tiempo solo se da donde hay movimiento, y este es, en todas sus variantes, un rasgo distintivo del reino físico. Habiendo señalado

${ }^{19}$ Aquí debemos tener presente una de las tesis de Aristóteles, a saber, que el tiempo es algo que se da siempre en relación con el movimiento, es decir, que "el tiempo es el número del movimiento según el antes y el después"; Phys. $\Delta$ 220a 24-25. Sigo la traducción de Ute Schmidt, Física, 2001, México, UNAM. Más adelante nos detendremos a analizar cómo se relaciona esta tesis con el problema que estamos abordando, pues si bien el tiempo físico solo se da en relación con el movimiento, la esencia de este y de aquel no son idénticas.

${ }^{20}$ Véase Vigo, op. cit., pp. 39-41. 
esto, veamos en qué consiste esta preexistencia de la sustancia, particularmente respecto de la generación y de la corrupción, y de qué modo se da.

Sin importar cuál sea la manera como algo llega a generarse, toda generación tiene tres elementos constitutivos: ser por obra o a causa de algo, desde algo y el algo que se genera. ${ }^{21}$ Este último algo, dice Aristóteles, está referido a cualquier categoría, esto es, el algo generado es algo determinado ya sea en sentido sustancial o en sentido accidental. Estos tres elementos constitutivos corresponden a la naturaleza (ser por obra de algo), a la materia (desde algo) y a la existencia concreta del compuesto (algo) sea esencial o no. Aún más significativo que decir que materia, forma y naturaleza son elementos constitutivos de la generación, es que Aristóteles, en el libro $\Lambda$, señala que las tres cosas, en el ámbito de lo físico, son sustancia: "Las substancias son de tres clases: la materia, que es algo determinado en apariencia (pues las cosas unidas por contacto y no por unión natural son materia y sujeto); la naturaleza, que es algo determinado y cierta manera de ser hacia la cual tiende la materia; y la tercera, compuesta de estas dos, es la individual, por ejemplo Sócrates o Calias". ${ }^{22}$ Para el Estagirita, el algo que se genera - el cual, en tanto ya generado, ya sea por obra de la naturaleza o por obra de la técnica, posee necesariamente materia- es aquello que puede ser nombrado como sustancia en grado sumo. ${ }^{23} \mathrm{Y}$

${ }^{21}$ La razón por la que hacemos de la generación y de la corrupción el modo ejemplar para comprender la primordialidad de la sustancia en cuanto al tiempo es la siguiente: los otros tipos de movimiento, la alteración, el crecimiento y el cambio de lugar ya presuponen que es algo lo que se altera, que es algo lo que crece, que es algo lo que cambia de lugar. Pero en el caso de la generación y la corrupción la situación es del todo distinta, pues ahí el movimiento que se da es el de que algo llega ser o algo deja de ser simplemente. Dicho de otro modo, en la generación hay paso del no-ser al ser, y viceversa, en sentido simple. Y puesto que se trata del no-ser como aquello desde lo cual algo llega a ser, y que en consecuencia sería primero en orden a la existencia de una sustancia, la cuestión que hay que aclarar es cómo ese no-ser no guarda una relación de anterioridad con la sustancia.

${ }^{22}$ Metaph. $\Lambda$ 1070a 9-13.

${ }^{23}$ Cfr. Metaph. Z 1032a 19. Esta tesis de que sustancia en grado sumo es lo concreto o individual se ajusta a lo dicho en Categorías, donde podemos leer lo siguiente: "Entidad [oủoía] la así llamada con más propiedad, más primariamente y en más alto grado, es aquella que, ni se dice de un sujeto, ni está en un sujeto, v.g.: el hombre individual o el caballo individual"; Cat. 2a 11-13. Sigo la traducción de Miguel Candel Sanmartín, Categorías, 2008, Madrid, Gredos. Ahora bien, en esta indicación es evidente que Aristóteles le da una preemi- 
la razón de ello reside en que el algo generado es aquello que, por principio, se encuentra en acto, esto es, se encuentra realizando su ser en el fin. Este estar en acto de lo generado significa que en el algo que ha llegado a ser reside ya la capacidad para poner en movimiento a otra cosa para que llegue a ser algo — ya sea naturalmente, o ya sea técnicamente - o bien para moverse a partir de sí mismo - lo cual solo se da en el caso de los vivientes - o bien para permanecer, en la medida de sus posibilidades, en su propio fin. Esto último, el permanecer de algo en su propio fin según sus posibilidades, se debe a que todos los entes generados poseen materia, y esta es precisamente aquello que funge como el principio a partir del cual las cosas tienen la potencia de ser o de no ser, tal y como podemos leer en el tratado Acerca de la generación y la corrupción: "causa en sentido de materia de los entes sujetos a generación es la "posibilidad de ser y no ser"'. ${ }^{24}$

Ahora bien, según lo que hemos dicho, materia, forma y naturaleza, en tanto que sustancias, deben preexistir en el orden del tiempo. Que la materia preexiste se debe a que en todo cambio, lo que cambia se da desde algo, y esto desde lo que algo cambia es precisamente un sustrato que es capaz de padecer el cambio, y esto es la materia. El cambio es una afección de la sustancia, y en tanto que tal, está necesitado de algo que sirva de sustrato en el que se padezca la generación y la corrupción. Por ejemplo, al cambiar una piedra de caliente a fría es necesario que preexista aquello que, estando afectado por lo caliente, puede ser afectado por el frío; de igual modo, cuando se genera una planta, es nece-

nencia a lo que es ente en virtud de una forma o especie que no se predica de otro ni se da en otro, razón por la cual los llamados accidentes no tendrían el carácter de entidad o sustancia. Sin embargo, debido a que para Aristóteles el algo generado puede abarcar a todas las categorías - aunque sea en un sentido analógico-, el significado que tiene el algo en este contexto, que se refiere a la concreción o individualidad de algo ente, no se limita al de las especies como la planta o el animal, sino que también se refiere a la cantidad, a la cualidad, etc. En el fondo, en esto no hay contradicción, pues si bien el accidente se puede generar como la sustancia, su generación se da según el orden del ser que funda la preeminencia de la oưoía en sentido estricto. Más adelante veremos cuál es la diferencia entre algo entendido como especie esencial - el cual se identifica en mayor medida con la acepción de oủoía empleada en Categorías - y el algo entendido como especie accidental, el cual está en dependencia de algo anterior, o mejor dicho, primordial en el orden de lo que es.

${ }^{24}$ Arist. De Gen. et Corr. 335a 32-33. Sigo la traducción de Ernesto Lacroce y Alberto Bernabé, De la generación y la corrupción, 1987, Madrid, Gredos. 
sario que preexista aquello desde lo que se hará la planta, a saber, cierto principio ínsito en la semilla que la potencia para que llegue a ser una planta, pero que también deja abierta la posibilidad de que no llegue a generarse la planta. Por ello dice Aristóteles: "La materia es, ante todo y en sentido propio, el sustrato capaz de recibir la generación y la corrupción, pero en cierto modo es también el sustrato de los otros tipos de cambio, porque todos los sustratos son capaces de recibir ciertas clases de contrariedad". ${ }^{25}$ Así, lo que preexiste es ese modo de ser que, siendo causa en algún sentido, subyace y mantiene abierta la posibilidad de ser y de no ser. Y esto es la materia.

Por otra parte, es necesario que la forma - ya sea en el sentido de accidentes, o sea ya en el sentido de especie- preexista en el tiempo y respecto del movimiento, porque si la forma no preexistiera, el cambio de las cosas procedería de la indeterminación y se encaminaría hacia la indeterminación, y lo que es más grave aún, no existiría propiamente el cambio. La razón de esto radica en que, como ya hemos señalado con Aristóteles, si solo preexiste la materia, y esta es aquello que por sí misma es indeterminada, y además no es un sujeto activo sino eminentemente pasivo, entonces al no preexistir algo que ponga en movimiento a la materia y la haga padecer sucedería que todo permanecería en la indeterminación y tampoco habría ningún tipo de movimiento. Pero esto contraviene a la evidencia de que, de hecho, el cambio se da en el ámbito de las sustancias sensibles. Por tanto, es necesario que exista un principio activo respecto de la materia y que, preexistiendo junto con la materia, sea capaz de hacer padecer el cambio en ella, esto es, que sea capaz de hacer que la materia llegue a ser algo determinado. Y esto es la forma, entendida en un sentido amplio. ${ }^{26}$ Que las

${ }^{25}$ Ibid., 320a 2-4.

${ }^{26} \mathrm{La}$ diferencia que establece Aristóteles entre la forma entendida como especie y la forma entendida como accidente reside en que esta última no preexiste en acto, sino solo en potencia, mientras que la forma como especie sí preexiste en acto. Así, los accidentes preexisten como aquello que necesariamente sobreviene como afección de una sustancia, por ejemplo, una mesa que está por producirse necesariamente habrá de tener cierta cualidad, cierta relación, cierto lugar. Una vez producida la mesa, y dada cierta situación, posee en acto tal cualidad, tal relación, y tal lugar: ser bella, ser de Pedro, estar en la casa. Por su parte, la forma en cuanto especie preexiste en acto en el alma del artesano en cuanto aquello con vistas a lo cual se ha de utilizar cierta materia para producir una cosa determinada, es decir, la especie de la mesa está 
formas preexisten se hace evidente por el hecho de que en todo cambio, lo que cambia siempre pasa de una forma a otra, es decir, siempre llega a ser algo determinado, pues aquello en vistas a lo cual se da el cambio es siempre un algo que es principio del ser de las cosas, ya sea en cuanto a los accidentes (alteración) ya sea en cuanto a la sustancia (generación y corrupción). ${ }^{27}$ De este modo, al iniciarse la generación de una casa, no solo preexisten la materia de la casa - es decir, la potencia para ser o no ser que se da en aquellas cosas que son aptas para emplearse en la construcción de la casa-, sino también la especie de esta en el alma del arquitecto. Cuando se ha concluido la generación, cuando la casa es en acto y existe, no se ha generado la forma de la casa ni su materia, sino que lo que se ha producido es esta casa, a partir de estos materiales, por obra del arquitecto. Y en el caso de los entes que se generan por naturaleza, como los animales, en estos también preexiste la forma y la materia: esta, en tanto que el semen y el óvulo son aquella materia desde la que puede generarse un animal, y aquella, la forma, preexiste en tanto que es aquello en vistas a lo cual se da la unión del semen y el óvulo - y que es la determinación fundamental de dicha unión-, a saber, este animal. Y así, finalmente, cuando se ha engendrado un animal, no se ha generado la especie animal ni su materia, sino este animal a partir de esta materia, esto es, lo que se genera es el compuesto de materia y forma. De este modo, la forma preexiste en cuanto al tiempo y respecto del movimiento en tanto que es ella el fin hacia el que se encamina toda generación, ya sea según arte, o bien sea según naturaleza.

de cierto modo ya en acto en el alma del artesano en tanto que es el principio que ha de determinar el ser de esta mesa elaborada a partir de tal materia. En el caso de los entes cuyo principio de movimiento es la naturaleza, el asunto no es muy distinto. La forma esencial, la especie, preexiste en acto, pero no en este ente que llega a generarse, sino en otro de la misma especie. Solo porque dicha forma esencial preexiste de ese modo es que puede llegar a generarse otro individuo de la misma especie. Pero lo que no preexiste en acto, sino en potencia, son los contenidos específicos del resto de las categorías que se llegan a corresponder con una sustancia. Así, cuando una planta llega a generarse, preexiste que pueda tener cualidad, pero no la actualidad efectiva de dicha cualidad, por ejemplo, el ser benéfica para alguien.

${ }^{27}$ Vigo, siguiendo a Wieland, también parece coincidir con este sentido eminentemente teleológico del movimiento, lo cual dotaría en todo punto de prioridad a la forma frente a la materia. A este respecto, véase "Prioridad y prioridad ontológica según Aristóteles". Estudios Aristotélicos, pp. 44-45. 
Ahora bien, uno podría preguntarse cómo es que la materia está dispuesta siempre a adquirir cierta forma, a llegar a ser algo determinado. La respuesta a esta pregunta la encontramos en la sustancia entendida como naturaleza. Ya nos ha dicho Aristóteles que la naturaleza es algo determinado y cierto modo de ser hacia el que tiende la materia. Ser algo determinado, o bien, principio del ser propio y del acto de las cosas es la forma. Por tanto, la naturaleza es la sustancia, o modo de ser, que se da en virtud del ensamble entre la materia y la forma, entre el acto y la potencia, entre la indeterminación y la determinación que da lugar a que, en el ámbito de las sustancias sensibles, la generación y la corrupción se den constantemente. Y por ello, la naturaleza es, propiamente, el sustrato último de todo cambio. Así, Aristóteles nos dice que, "en general, aquello desde lo que se genera y según lo que se genera es naturaleza (pues lo que es generado tiene naturaleza, por ejemplo una planta o un animal), y aquello por obra de lo cual se genera es la naturaleza llamada específica, que es de la misma especie (pero esta está en otro); pues un hombre genera a un hombre". ${ }^{28}$ Esto no contraviene al hecho de que exista un modo de generación a partir del arte, pues si bien el modo de generarse cosas según arte es a partir de aquello que no tiene dentro de sí el principio de movimiento, no obstante lo que es principio de movimiento en el arte sí es un ente natural, es decir, es un ente que posee dentro de sí el principio de movimiento y que posee la capacidad de mover de tal modo, productivamente, a otros entes. ${ }^{29}$ De este modo, la naturaleza, tanto

${ }^{28}$ Metaph. Z 1032a 23-25.

${ }^{29}$ Aquí no debemos perder de vista lo que Aristóteles afirma en la Física acerca de la

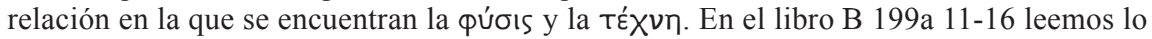
siguiente: "Por ejemplo: si una casa perteneciera a los objetos que se dan por naturaleza, se produciría, como ahora, por un arte; pero si las cosas naturales se produjeran no solo por naturaleza, sino también por arte, se producirían del mismo modo como se hacen por naturaleza. Por tanto, lo uno existe en vista de lo otro. En general el arte termina de ejecutar en algunos casos lo que la naturaleza no es capaz de completar, pero en otros, la imita". Se pueden hacer todos los matices que se quieran para diferenciar arte y naturaleza, pero las estructuras definitorias de una y otra son semejantes en alto grado: ambas convergen en el a partir de (materia) y en el con vistas a algo (forma); se distinguen en el modo específico en el que se da el ensamble de materia y forma. Pero justo ahí tiene peso lo que dice Aristóteles: arte y naturaleza son el uno para el otro, en correspondencia, no como principios que se encaminen a fines distintos. Solo por ello puede el arte completar lo que la naturaleza no logra por sí, o bien puede imitarla. 
en general como específica, abarca y determina de cierto modo a todo lo que puede llegar a ser en el ámbito de las sustancias sensibles. Y en tanto que las determina, y en tanto que la naturaleza es el ensamble entre el acto y la potencia, entre la materia y la forma, ella misma es también primordial en cuanto al tiempo y respecto del movimiento. Esta primordialidad radica en que en ella está contenido el principio que rige a todo lo que existe en el ámbito sensible, el cual Aristóteles expresa del siguiente modo:

En efecto, dado que afirman que en todas las cosas la naturaleza aspira a lo mejor, y que es mejor ser que no ser [...] pero es imposible que el ser esté presente en todas las cosas debido a lo muy lejos que se encuentran del principio, el dios consumó el universo en el único modo que le restaba, haciendo ininterrumpida la generación. Pues así el ser puede poseer el mayor grado de consistencia, gracias a que el perpetuo producirse de la generación es lo más cercano que hay a la sustancia. ${ }^{30}$

\section{Primordialidad de la sustancia en cuanto al tiempo}

En nuestros análisis anteriores hemos perfilado en qué sentido la sustancia tiene una primordialidad en cuanto al movimiento - pensado en su forma más radical, como generación y corrupción- y por ende, en cuanto al tiempo. Aquí no debemos pasar por alto que, como dice Aristóteles, "es claro que el tiempo no es movimiento, pero tampoco se da $\sin$ movimiento" ${ }^{31}$ y por ello, si la sustancia tiene una relación

${ }^{30}$ De Gen. et Corr. 338b 27-30.

${ }^{31}$ Phys. $\Delta 219$ a 1-2. El ejemplo en el que se basa Aristóteles para sostener esta tesis es sumamente interesante. En vez de apelar a un tipo de cambio externo, el Estagirita se refiere al pensamiento y destaca que cuando nosotros no cambiamos nuestros pensamientos, o hemos pasado por alto hacerlo, entonces nos parece que no ha transcurrido tiempo. Se trata de una especie de insensibilidad para el cambio, y por ende, para el tiempo. A partir de esto, Aristóteles extrae una observación relevante: si el ahora anterior y el posterior nos aparecen como el mismo - es decir, como si no hubiese cambio alguno entre uno y otro ahora-, entonces no hay posibilidad de sentir el tiempo. En este sentido, cabe decir que si existe una forma de ser cuyo ahora sea siempre el mismo, entonces dicha forma de ser no es temporal, y no solo en el sentido de que no sea corruptible, sino también en el sentido de que su existencia se mueve en un plano distinto al del tiempo. Por otro lado, y esto es evidencia incontrovertible para nuestro autor, la modificación de lo que se da entre dos determinados ahora, uno anterior y otro posterior, es una señal que nos incita a sentir el tiempo. 
de primordialidad respecto del movimiento, y a una con ello, el tiempo presupone alguna clase de movimiento para existir, entonces resulta claro que la sustancia es también primordial respecto del tiempo. Lo que se debe aclarar en este punto es cómo se da dicha primordialidad según la estructura del tiempo.

Con todo, quizás quepa hacer una última aclaración antes de continuar con nuestro cometido. Se trata del lugar preeminente que le damos a la qúoıs, y no simplemente a la forma. Que afirmemos que es la naturaleza lo que preexiste como sustancia en el orden de lo sensible - el cual abarca todo lo que es, a excepción quizás del primer motorse debe a que es el nexo necesario para que la materia pueda alcanzar una forma definida. Este nexo de forma y materia, la naturaleza, ha existido siempre. Es impensable, por otro lado, que la forma y la materia hayan existido alguna vez separadas dada la eternidad del mundo. Pero no solo esto. También sucede que en la naturaleza, en su propio modo de ser, está ínsita la tendencia y la tensión necesaria que hace que lo ente goce del mayor grado de consistencia posible; sin la 申úoıs sería imposible en todo punto que la materia alcanzase algún tipo de determinación, a la vez que sería imposible que la forma adquiriese una existencia efectiva. Además, si la forma, dentro del ámbito sensible, fuese lo preexistente en sentido absoluto, entonces no habría movimiento alguno en dicho ámbito, y esto es algo que contraviene a la evidencia que los hechos ponen de manifiesto. Фúoıs es la manera de ser de la sustancia con la que nos hallamos inmediata y regularmente, y en medio de la cual se realiza todo existir. Así, debemos decir que, dentro de los límites que hemos fijado en nuestras descripciones, la distinción entre forma y materia es una distinción analítica, y esto quiere decir que se trata de una distinción entre los elementos que pertenecen a una unidad previa que ensambla y delimita las posibilidades de ser de aquellas. En apoyo a esta tesis podemos apelar al sentido primario que indica Aristóteles para la sustancia en su tratado de Categorías 2a, 11-13. A esta afirmación no se le puede contraponer aquella tesis de que la forma es anterior respecto de la materia y de la naturaleza, en tanto que es la condición que aporta la determinación última de la existencia de algo. En todo caso, las formas, tomadas por separado, serían 
como una especie de cualidad, esto es, una sustancia segunda. Esta tesis de la prioridad de la forma es válida desde el punto de vista de aquello que produce la definición del contenido de un ente, pero no desde el punto de vista correspondiente a las condiciones de existencia de una cosa, pues en este caso son necesarias tanto la materia como la forma, y no como una mera aglomeración, sino como elementos que se pertenecen de manera irrenunciable. A esta pertenencia mutua, que se manifiesta en la existencia de un algo concreto, es a lo que llamamos primordialidad de la púoıs.

Una vez aclarado lo anterior, nos hacemos la siguiente pregunta: ¿en qué consiste la primordialidad de la sustancia según la estructura del tiempo? Para responder, debemos reiterar que el marco en el que se mueve dicha pregunta es algo bien delimitado, a saber, el ámbito de la llamada sustancia sensible. Esto no es de poca importancia en la medida en que tanto el movimiento como el tiempo solo se dan en dicho ámbito. No obstante, su modo de darse es distinto, y nos lo señala Aristóteles así: "Por un lado el cambio y el movimiento de cada [cosa] se dan solo en lo que cambia o donde por suerte se encuentra la cosa que se mueve y que cambia. Por otro lado, el tiempo se encuentra igualmente en todas partes y en todas las [cosas]". ${ }^{32}$ El movimiento solo se da en algo - sea al modo de crecimiento, de alteración o de corrupcióno en el lugar en el que algo se encuentra — movimiento local—, mientras que el tiempo atraviesa a todo y todos los lugares, al menos como una posibilidad. Esto no contraviene a la dependencia del tiempo respecto del movimiento, en tanto que sin el cambio en cualquiera de sus formas no podríamos señalar tiempo alguno; se trataría del mismo "ahora" que no señala ninguna transición de un antes hacia un después, sino de un "ahora" que acusa una presencia simple. Así, de lo que se trata es de poner a la vista, suponiendo el movimiento, en qué consiste eso de que, al menos como posibilidad, el tiempo esté en todo y en todas partes; por otro lado, debemos mostrar cómo esa omnipresencia del tiempo está atravesada por la primordialidad de la sustancia. Así, preguntamos: ¿cómo es aquella posibilidad de que el tiempo esté en todo y en todas partes?

\footnotetext{
${ }^{32}$ Phys. $\Delta$ 218b 10-14.
} 
La omnipresencia del tiempo depende que alguno de sus elementos tenga una existencia concreta. Lo decimos de este modo porque se nos presenta la siguiente dificultad: ¿qué existe del tiempo, si lo sido ya no es y lo que será aún no es? Así, es preciso que algo del tiempo exista, y que en consecuencia sea cognoscible. A esa parte del tiempo que existe de hecho y que es cognoscible de modo inmediato es lo que Aristóteles llama ahora ( $v \tilde{v} v)$. La afirmación de nuestro autor no deja lugar a equívocos: "Es claro también que si no hubiera el tiempo, tampoco habría el ahora, y si no hubiera el ahora, tampoco habría tiempo". ${ }^{33}$ Y es justo a partir del estudio de la manera de ser del ahora que el Estagirita puede señalar cómo es que el tiempo está en todo y en todas partes. En un sentido, el ahora es siempre diferente puesto que se puede decir en referencia a distintos movimientos, y visto así, la existencia concreta de esa ahora siempre puede ser distinta. Así, ahora mismo puedo decir que escribo, y de este modo, el contenido de ese ahora es mi acto de escribir; sin embargo, si posteriormente en vez de escribir lo que hago es deliberar, entonces existe el ahora con un contenido distinto, el de mi acto de deliberación. En otro sentido, el ahora es siempre lo mismo en tanto que mientras es, su ser no radica en otra cosa más que señalar la presencia actual de algo en movimiento, y a una con ello, limita y distingue entre lo anterior y lo posterior dentro del cambio de esa misma cosa. El ahora es el mismo en tanto que sigue a la unidad de lo que se mueve, es decir, es $s u$ ahora en un determinado momento del movimiento, y a fuer de tal, es idéntico con eso que ahora se mueve. De esta manera, sucede como con el número, que siendo idéntico en su esencia, puede estar en distintas cosas a la vez; así como decimos aquí hay un vaso, allá una ventana, de igual modo podemos decir ahora escribo, ahora escucho un avión.

Hasta aquí, podemos decir que la condición para ser del tiempo, el ahora, existe como el momento de presencia de una cosa en movimiento, como ese momento que limita entre lo anterior y posterior. Ese momento de presencia es el mismo en todo y en todas partes, su manera de ser es la misma - limitando y diferenciando entre lo anterior y lo

${ }^{33}$ Ibid., $\Delta$ 219b 33-220a 1. Las cursivas son mías. 
posterior-; pero a la vez distinto por lo que respecta a su concreción última, la de este ahora, el cual singulariza a la vez lo que llegue a ser la concreción de este anterior y este posterior. Solo porque el ahora es así, puede el tiempo estar en todos lados, en todas las cosas. Pero no olvidemos que esto, el ahora, no es aún el tiempo, sino solo su condición existente más cognoscible.

En atención al otro aspecto de la relación ahora-tiempo, esa que dice que el ahora no es sin tiempo, cabe decir que es como la presencia de algo en movimiento, esto es, pertenece a algo en lo que puede desplegar su propia esencia. El ahora solo puede ser todo lo que es en el tiempo; la presencia en el movimiento. Esto quiere decir que a todo ahora le pertenece una referencia a lo anterior y a lo posterior, y solo ahí existe el ahora, como unidad de medida de ese movimiento de lo uno a lo otro. Es en el tiempo en donde efectivamente pueden ser el ahora, lo anterior y lo posterior; no existen por sí mismos sino en tanto que son algo del tiempo. El tránsito que vemos del movimiento de una cosa proviene de algo y marcha hacia algo; su presencia cambiante así lo indica. Pero de igual modo, tanto la presencia en tránsito, así como el desde algo y el hacia algo son algo del movimiento. El ahora es la unidad de conteo, de medida de la duración de ese tránsito delimitado de algo a algo, de lo anterior a lo posterior. El ahora está en el tiempo, y este es el número del movimiento, de la duración, dentro del límite entre lo anterior y lo posterior. De lo anterior se sigue la definición que Aristóteles da al tiempo: "el número del movimiento en relación con lo 'antes' y lo 'después'". ${ }^{34}$ En el tiempo está todo movimiento posible en tanto que es medido en su duración en virtud del ahora.

Pues bien, considerando este doble aspecto del modo de ser del $v \tilde{v} v$, y su pertenencia al tiempo, se puede decir que el tiempo está en todas partes y en todas las cosas - las que son susceptibles de cambio, en cualquiera de sus sentidos- - Y es desde esta perspectiva que se debe resaltar la primordialidad de la sustancia según el tiempo. En este sentido, cabe decir que si hay una cosa cuyo ahora, cuya presencia sea siempre la misma, entonces esa cosa no es temporal. Pero respecto de una

${ }^{34}$ Ibid., $\Delta$ 219b 2-3. 
cosa tal no vale la primordialidad de la sustancia según el tiempo. Primordial no puede significar algo que es anterior al tiempo, puesto que visto así, lo que es anterior al tiempo no está en el orden de este. Ser primordial según el tiempo quiere decir ser lo primero en el orden del tiempo, no más allá de él. Así, podemos preguntarnos: ¿qué es aquello del tiempo que nos da una señal acerca de la primordialidad de la sustancia? A nuestro parecer, una indicación de la primordialidad de la sustancia se encuentra en el ahora, que se refiere a la presencia de algo en movimiento o susceptible de moverse. El ahora apunta a aquello que cambia, hacia aquello que dura. Y lo que cambia o dura es una sustancia, o bien una afección de la sustancia. En primera y última instancia, es la existencia de la sustancia, actual o potencialmente, la que permite numerar el movimiento de algo hacia algo. Por ello es que el movimiento de cualquier cosa, y su tiempo, son algo continuo, pues aquello a lo que siguen en general el tiempo y el movimiento es a la sustancia. Ella es el límite primero y último de todo cambio, de todo tiempo; entretanto, se pueden señalar cualesquiera momentos de la sustancia, cualidades, relaciones, pero todas penden de ese límite que es la presencia de la sustancia. Por ejemplo, si tomamos el día como sustancia, al decir que han ocurrido tres días, con ello nombramos el movimiento de los días en un determinado lapso de tiempo, desde ese ahora que era hace tres días hasta este ahora actual, hasta este día. Sin el contenido de la presencia a partir del ahora, que no es más que la sustancia en movimiento señalable en el horizonte lo anterior y lo posterior, no hay posibilidad de que se dé el tiempo. Así, la sustancia es primordial según el tiempo por ser ella lo correspondiente, en última instancia, a aquella parte del tiempo que existe en grado sumo y que es la más cognoscible, el ahora.

\section{Conclusión}

A modo de conclusión, podemos decir que, en Aristóteles, el concepto de lo primordial sirve para señalar los problemas centrales a los que se enfrenta la filosofía primera, con miras a la constitución de una ciencia cuyo asunto sea el saber fundamental acerca de la totalidad de lo ente, 
el cual solo es posible si se tiene en claro qué es la sustancia y lo que le corresponde de suyo. Estos problemas fundamentales de la filosofía primera son: el problema de la definición, el del conocimiento y el del tiempo. Si bien en todos estos casos la sustancia es lo primordial, debe dejarse en claro por qué lo es y qué implicaciones tiene esto en cada

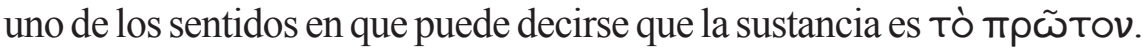
Por lo que respecta al contenido de un ente, podemos decir que sustancia en sentido eminente es la forma entendida como especie, pues solo ella puede ser definida, conocida y es aquello que antecede como fin a todo cambio y lo que en él puede ser numerado según lo anterior y lo posterior. Sin embargo, esta eminencia de la especie o de la forma debe ser pensada principalmente según la primordialidad en cuanto al tiempo - es decir, en el sentido de lo primordial que es la sustancia en cuanto naturaleza - pues como ha dicho Aristóteles, la naturaleza es ese modo de ser que más se asemeja a lo que la sustancia propiamente es, y porque por otra parte, hemos señalado que en ella se ensamblan la materia y la forma, la potencia y el acto. Aquí sigue siendo cuestionable si el criterio para definir la preeminencia de la forma respecto de la naturaleza es una gradación del ser, puesto que, en efecto, el movimiento tiende siempre a una forma, y esto parece denotar una mayor perfección en aquello hacia lo que se tiende.

Sin embargo - y esto es lo relevante — en el ámbito natural, la forma realizada no es un límite último, sino que esa forma ligada a la materia sigue siendo corruptible, y por ello no está exenta de poder-ser. De este modo, resta por pensar cómo se relaciona la eminencia de la forma en cuanto al contenido con la primordialidad de la sustancia entendida como naturaleza. De ello depende qué y cómo sea el sentido teleológico del universo, el cual Aristóteles acepta. Una vez aclarado esto, se debería mostrar qué relación guarda lo anterior con otros dos problemas: por una parte, por qué el acto es anterior a la potencia, es decir, por qué es más ente lo que es que lo que puede ser o no-ser; y por otra, por qué lo que Aristóteles considera como sustancia en sentido propio - a saber, el primer motor inmóvil, Dios - es aquello que está separado de todas las cosas. Esto, llevado a sus últimas consecuencias, equivale 
a preguntarse cuál es la relación que sostiene el tiempo con la eternidad de la sustancia absoluta.

Finalmente, también queda abierta la pregunta de qué lugar ocupa la naturaleza dentro de la multiplicidad de sentidos en los cuales se dice la sustancia, que es el tema de Metafísica Z. Esto quiere decir que se debe aclarar en qué medida la primordialidad de la sustancia entendida como naturaleza puede desanudar las aporías que quedan fincadas en las investigaciones realizadas por Aristóteles. En todo caso, el Estagirita no parece decidirse por el sujeto, por la esencia, por el género o por el universal como sentido definitorio de la sustancia, pues ninguna de ellas cumple a cabalidad con los criterios de primordialidad establecidos por él mismo. Sin embargo, la sustancia entendida como naturaleza, ya sea en general o como naturaleza específica, sí que se presenta como una forma de ser primordial en los tres órdenes, considerando ante todo el peso de la afirmación de Aristóteles en el capítulo segundo de Categorías. Con esta interpretación queda sugerido que buena parte del trabajo de la ontología ha de darse, como ya lo sabía el propio Aristóteles, bajo la pregunta de qué es la naturaleza. 
CITAM Derechos Reservados.

La reproducción total o parcial de este artículo se podrá hacer si el ITAM otorga la autorización previamente por escrito. 